\title{
Additive functions with respect to expansions over the set of Gaussian integers
}

\author{
by \\ I. KÁtai (Budapest) and P. Liardet (Marseille)
}

\section{Introduction}

1.1. Let $\mathbb{Z}[i]$ be the ring of Gaussian integers, $\theta \in \mathbb{Z}[i]$ such that $t:=$ $|\theta|^{2} \geq 2$, and $\mathcal{A}=\left\{a_{0}=0, a_{1}, \ldots, a_{t-1}\right\}(\subseteq \mathbb{Z}[i])$ a complete residue system $\bmod \theta$. We call $\mathcal{A}$ the set of digits. Then, for each $\alpha \in \mathbb{Z}[i]$, there exists a unique $\alpha_{1} \in \mathbb{Z}[i]$ and a unique $b_{0} \in \mathcal{A}$ such that $\alpha=b_{0}+\theta \alpha_{1}$. The function $J: \mathbb{Z}[i] \rightarrow \mathbb{Z}[i]$ is defined by $J(\alpha)=\alpha_{1}$.

Iterating $J$, we define the orbit

$$
\alpha\left(=\alpha_{0}\right), \quad \alpha_{1}=J\left(\alpha_{0}\right), \quad \alpha_{2}=J\left(\alpha_{1}\right), \ldots
$$

Let

$$
L:=\frac{1}{|\theta|-1} \max _{a \in \mathcal{A}}|a|
$$

It is easy to show that

(a) if $|\alpha|>L$, then $\left|\alpha_{1}\right|<|\alpha|$,

(b) if $|\alpha| \leq L$, then $\left|\alpha_{1}\right| \leq L$.

Hence the orbit (1.1) is ultimately periodic for every $\alpha \in \mathbb{Z}[i]$. The proof of the two easy assertions stated above is given in the lecture notes [3].

An integer $\pi \in \mathbb{Z}[i]$ is said to be periodic if there is a positive integer $k$ for which $J^{k}(\pi)=\pi$. Let $\mathcal{P}$ be the set of periodic points. From the assertions (a) and (b) we see that if $\pi \in \mathcal{P}$, then $|\pi| \leq L$.

Repeating the expansion defined above, we obtain

$$
\alpha=b_{0}+b_{1} \theta+\ldots+b_{k-1} \theta^{k-1}+\theta^{k} \alpha_{k} \quad(k=0,1, \ldots),
$$

where the sequence of the digits $b_{0}, \ldots, b_{k-1}$ is uniquely determined by $\alpha$ and $\theta$. Let $k$ be the smallest nonnegative integer for which $\alpha_{k} \in \mathcal{P}$. Then (1.2) with this $k$ is called the correct expansion of $\alpha$. By this convention,

2000 Mathematics Subject Classification: 11A63, 11K65. 
each $\alpha$ has a unique correct expansion. Let $l(\alpha):=k$ be the length of the representation. Then $l(\alpha)=0$ if and only if $\alpha \in \mathcal{P}$.

A system $(\theta, \mathcal{A})$ is called a number system (or a numeration system) if $\mathcal{P}=\{0\}$. In that case each $\alpha \in \mathbb{Z}[i]$ has a finite expansion.

In [4] it was proved that $\mathcal{A}=\{0,1, \ldots, t-1\}$ is an appropriate digit set for $\theta$ to generate a number system if and only if it has the form $\theta=-A+i$ or $\theta=-A-i$ with $A \geq 1$. G. Steidl [6] proved that for $\theta \in \mathbb{Z}[i]$ there is a suitable digit set $\mathcal{A}$ such that $(\theta, \mathcal{A})$ is a number system if and only if $t \geq 2$ and $1-\theta$ is not a unit.

1.2. Assume that $\theta, \mathcal{A}$ are fixed.

Definition 1. A function $f: \mathbb{Z}[i] \rightarrow \mathbb{R}$ is additive (with respect to the expansion generated by $\theta$ and $\mathcal{A}$ ) if

(a) $f\left(\pi \theta^{k}\right)=0$ for $\pi \in \mathcal{P}$ and $k=0,1, \ldots$,

(b) for every $\alpha \in \mathbb{Z}[i]$,

$$
f(\alpha)=f\left(b_{0}\right)+f\left(b_{1} \theta\right)+\ldots+f\left(b_{k-1} \theta^{k-1}\right),
$$

where $\alpha=b_{0}+b_{1} \theta+\ldots+b_{k-1} \theta^{k-1}+\theta^{k} \pi$ is the correct expansion of $\alpha$.

Let $\mathcal{E}_{\theta}$ be the class of additive functions in the above sense.

Definition 2. A function $g: \mathbb{Z}[i] \rightarrow \mathbb{C}$ is multiplicative (with respect to the expansion generated by $\theta$ and $\mathcal{A}$ ) if

(a) $g\left(\pi \theta^{k}\right)=1$ for $\pi \in \mathcal{P}$ and $k=0,1, \ldots$,

(b) for every $\alpha \in \mathbb{Z}[i]$,

$$
g(\alpha)=\prod_{j=0}^{k-1} g\left(b_{j} \theta^{j}\right) .
$$

Let $\mathcal{M}_{\theta}$ be the class of multiplicative functions in the above sense. Let $\overline{\mathcal{M}}_{\theta} \subseteq \mathcal{M}_{\theta}$ be the set of those $g$ for which additionally $|g(\alpha)|=1$ for all $\alpha \in \mathbb{Z}[i]$.

1.3. Since

$$
\frac{|\alpha|-K}{|\theta|} \leq|J(\alpha)| \leq \frac{|\alpha|+K}{|\theta|}
$$

where

$$
K=\max _{a \in \mathcal{A}}|a|,
$$

iterating we get the following

Lemma 1. There exist suitable positive constants $c_{1}, c_{2}$ (depending on $\theta$ and $K$ ) such that

$$
-c_{2}<l(\alpha)-\frac{\log |\alpha|}{\log |\theta|}<c_{1} \quad \text { for every } \alpha \in \mathbb{Z}[i] \backslash\{0\} .
$$


2. Formulation of the main results. Our purpose in this paper is to give necessary and sufficient conditions for the existence of the mean value of $g \in \overline{\mathcal{M}}_{\theta}$, where the summation is extended to a disc around zero with growing radius, or to some sectors of it.

We shall prove that the analogue of Delange's theorem for $q$-multiplicative functions [1] remains valid (see Theorem 1). As an application we give necessary and sufficient conditions for the existence of the limit distribution of $f \in \mathcal{E}_{\theta}$ (see Theorem 2). Finally we prove a theorem for the local distribution of the sum of digits function (see Theorem 4).

3. Lemmata. For an interval $I \subseteq[-1 / 2,1 / 2)$ let $C_{I}$ denote the annulus $\{z|z \in \mathbb{C}, 1 /| \theta|<| z \mid<1,(\arg z) /(2 \pi) \in I\}$. For $g \in \overline{\mathcal{M}}_{\theta}$ let

$$
S_{I}(x \mid g):=\sum_{\alpha \in x C_{I}} g(\alpha),
$$

where $x$ is a positive growing parameter and $x C_{I}=\left\{x z \mid z \in C_{I}\right\}$.

It is well known that $S_{I}(x \mid 1)=$ number of Gaussian integers in $x C_{I}$ is $\pi|I| x^{2}(1-1 / t)+O(x)$ as $x \rightarrow \infty$, uniformly in $I$.

Let

$$
\begin{aligned}
N_{x} & :=\frac{\log x}{\log |\theta|}, \\
\Delta_{j} & :=\sum_{b \in \mathcal{A}} g\left(b \theta^{j}\right) .
\end{aligned}
$$

Lemma 2. Assume that $g\left(b \theta^{j}\right) \rightarrow 1$ as $j \rightarrow \infty, b \in \mathcal{A}$. Then there is a monotonic sequence $R_{N} \rightarrow \infty$ of positive integers such that

$$
\max _{|\beta| \leq|\theta|^{R_{N}}}\left|1-g\left(\beta \theta^{N}\right)\right| \rightarrow 0 \quad(N \rightarrow \infty) .
$$

Proof. Clear.

Let $\Gamma_{k}$ be the set of those Gaussian integers which can be written as $b_{0}+b_{1} \theta+\ldots+b_{k-1} \theta^{k-1}$, where the $b_{\nu}$ run over the set $\mathcal{A}$. Then $\Gamma_{k}$ is a complete residue system $\bmod \theta^{k}$. For $\alpha \in \mathbb{Z}[i]$ let $s_{k}(\alpha)\left(\in \Gamma_{k}\right)$ be defined by $\alpha \equiv s_{k}(\alpha)\left(\bmod \theta^{k}\right)$.

Lemma 3. Assume that $g\left(b \theta^{j}\right) \rightarrow 1$ as $j \rightarrow \infty$, for all $b \in \mathcal{A}$. Then there exists an increasing sequence of integers $M_{x}<N_{x}$ such that $N_{x}-M_{x} \rightarrow$ $\infty, M_{x} \rightarrow \infty$, for which

$$
g(\alpha)=\left(1+o_{x}(1)\right) g\left(s_{M_{x}}(\alpha)\right) \quad(x \rightarrow \infty)
$$

uniformly as $x /|\theta| \leq|\alpha| \leq x$; furthermore

$$
S_{I}(x \mid g)=\left(1+o_{x}(1)\right)|I|\left(1-\frac{1}{t}\right) \pi x^{2} \prod_{j=0}^{M_{x}-1} \frac{1}{t} \Delta_{j}+o\left(x^{2}\right) .
$$


Proof. The first assertion is a direct consequence of Lemma 2. Let $\alpha=$ $s_{M_{x}}(\alpha)+\theta^{M_{x}} \alpha_{M_{x}}$. Then

$$
\left|\alpha_{M_{x}}\right| \leq \frac{x}{|\theta|^{M_{x}}}+\frac{\left|s_{M_{x}}(\alpha)\right|}{|\theta|^{M_{x}}} \leq|\theta|^{N_{x}-M_{x}+1}+\frac{K}{|\theta|-1}<|\theta|^{N_{x}-M_{x}+c}
$$

with some constant $c>0$. By taking $M_{x}=N_{x}+c-R_{N_{x}}$ we find that $g\left(\alpha_{M_{x}} \theta^{M_{x}}\right) \rightarrow 1$ uniformly in the domain, thus the first assertion is proved.

Thus we have

$$
S_{I}(x \mid g)=\sum_{\alpha \in x C_{I}} g\left(s_{M_{x}}(\alpha)\right)+o(1) x^{2}|I| .
$$

To evaluate the sum on the right hand side, we write $\alpha$ as $\alpha=\beta+\theta^{M_{x}} \gamma$, where $\beta \in \Gamma_{M_{x}}$. If $\gamma \in \mathbb{Z}[i]$ occurs as a component of some $\alpha$ in $x C_{I}$, then

$$
\begin{aligned}
& \frac{x}{|\theta|^{M_{x}+1}}-\frac{K}{|\theta|-1} \leq|\gamma| \leq \frac{x}{|\theta|^{M_{x}}}+\frac{K}{|\theta|-1}, \\
& \left|\arg \gamma-M_{x} \arg \theta\right|<\frac{c|\theta|^{M_{x}}}{\alpha}<c \cdot|\theta|^{M_{x}-N_{x}} .
\end{aligned}
$$

For all but $O\left(x /|\theta|^{M_{x}}\right)$ of $\gamma$ satisfying (3.5) and (3.6) all of the integers $\beta+\theta^{M_{x}} \gamma, \beta \in \Gamma_{M_{x}}$, belong to $x C_{I}$. Since the number of Gaussian integers in the domain defined by (3.5), (3.6) is

$$
\pi|I|\left(\frac{x}{|\theta|^{M_{x}}}\right)^{2}+O\left(\frac{x}{|\theta|^{M_{x}}}\right)
$$

we have

$$
S_{I}(x \mid g)=\pi|I|\left(\frac{x}{|\theta|^{M_{x}}}\right)^{2}\left(1-\frac{1}{t}\right) \sum_{\beta \in \Gamma_{k}} g(\beta)+O\left(x|\theta|^{M_{x}}\right)+o(1)\left(x^{2}|I|\right) .
$$

Since $|\theta|^{M_{x}} \ll x|\theta|^{M_{x}-N_{x}}$ and $\sum_{\beta \in \Gamma_{k}} g(\beta)=\prod_{j=0}^{M_{x}-1}(1 / t) \Delta_{j}$, (3.4) immediately follows.

Lemma 4. Let $g \in \overline{\mathcal{M}}_{q}$. Assume that there exists a constant $c>0$, an infinite sequence $0 \leq l_{1}<l_{2}<\ldots$ of integers and a suitable sequence of digits $b_{1}, b_{2}, \ldots \in \mathcal{A}$ such that $\left|1-g\left(b_{\nu} \theta^{b_{\nu}}\right)\right| \geq c(\nu=0,1, \ldots)$. Then

$$
\frac{S_{I}(x \mid g)}{S_{I}(x \mid 1)} \rightarrow 0 \quad(x \rightarrow \infty)
$$

uniformly for every interval I whose length is bounded below by a positive constant.

Proof. We argue as in the proof of Lemma 3. Let $M_{x}$ be so chosen that $N_{x}-M_{x} \rightarrow \infty$ slowly. Let us write each $\alpha \in x C_{I}$ as $\beta+\theta^{M_{x}} \gamma$. Then

$$
S_{I}(x \mid g)=\sum g\left(\theta^{M_{x}} \gamma\right) \Sigma_{\gamma}
$$


where $\Sigma_{\gamma}$ is the sum of $g(\beta)$ over those $\beta \in \Gamma_{M_{x}}$ for which $\beta+\theta^{M_{x}} \gamma \in x C_{I}$. Thus

$$
S_{I}(x \mid g) \leq \sum_{\gamma}\left|\Sigma_{\gamma}\right| .
$$

We have $\left|\Sigma_{\gamma}\right| \leq t^{M_{x}}$, and

$$
\Sigma_{\gamma}=\prod_{j=0}^{M_{x}-1} \Delta_{j}
$$

if $\beta+\theta^{M_{x}} \gamma \in x C_{I}$ for every $\beta \in \Gamma_{M_{x}}$. Hence we obtain

$$
\left|S_{I}(x \mid g)\right| \leq c x^{2} \prod_{j=0}^{M_{x}} \frac{1}{t}\left|\Delta_{j}\right|+O\left(x|\theta|^{M_{x}}\right) .
$$

To finish the proof it is enough to observe that $(1 / t)\left|\Delta_{j}\right|<1-\delta(c)$ with some positive constant $\delta(c)$ depending on $c$, if $j \in\left\{l_{\nu}\right\}_{\nu=1}^{\infty}$. This is a direct consequence of the following

Lemma 5. Let $\omega_{0}, \ldots, \omega_{t-1}$ be complex numbers of modulus $1, \omega_{0}=1$, and $\Delta:=\omega_{0}+\ldots+\omega_{t-1}$. Then

$$
t^{2}-|\Delta|^{2} \geq \sum_{j=1}^{t-1}\left|1-\omega_{j}\right|^{2} .
$$

Proof. It is enough to observe that $2 \operatorname{Re}\left(1-\omega_{j}\right)=\left|1-\omega_{j}\right|^{2}$. From the identity

$$
t^{2}-|\Delta|^{2}=2 t \sum \operatorname{Re}\left(1-\omega_{j}\right)-\left|\sum\left(1-\omega_{j}\right)\right|^{2},
$$

and from the Hölder inequality

$$
\left|\sum_{j=1}^{t-1}\left(1-\omega_{j}\right)\right|^{2} \leq(t-1) \sum\left|1-\omega_{j}\right|^{2}
$$

the assertion immediately follows.

4. Consequences. We are ready to formulate our result.

Theorem 1. Let $g \in \overline{\mathcal{M}}_{\theta}$.

(1) If the series

$$
\sum_{j=0}^{\infty} \sum_{c \in \mathcal{A}} \operatorname{Re}\left(1-g\left(c \theta^{j}\right)\right)
$$

is divergent, then

$$
\frac{S_{I}(x \mid g)}{S_{I}(x \mid 1)} \rightarrow 0 \quad \text { as } x \rightarrow \infty
$$


uniformly on the intervals I whose length is bounded below by a positive constant. Consequently,

$$
\frac{1}{\pi x^{2}|I|} \sum_{\substack{|\alpha| \leq x \\ \arg \alpha \in I(\alpha)}} g(\alpha) \rightarrow 0 .
$$

(2) If (4.1) is convergent, then

$$
\lim _{x \rightarrow \infty}\left|\frac{S_{I}(x \mid g)}{S_{I}(x \mid 1)}\right|=\prod_{j=0}^{\infty} \frac{1}{t}\left|\Delta_{j}\right|,
$$

and the right hand side is non-zero if and only if $\Delta_{j} \neq 0(j=0,1, \ldots)$.

(3) The non-zero limit

$$
\lim _{x \rightarrow \infty} \frac{S_{I}(x \mid g)}{S_{I}(x \mid 1)} \quad(=m)
$$

exists if and only if

$$
\sum_{j=0}^{\infty} \sum_{c \in \mathcal{A}}\left(1-g\left(c \theta^{j}\right)\right)
$$

is convergent, and $\Delta_{j} \neq 0(j=0,1, \ldots)$.

Proof. If (4.1) is divergent, then by Lemma $5, \sum\left(1-(1 / t)\left|\Delta_{j}\right|\right)=\infty$, and so $\prod_{j=0}^{M_{x}}(1 / t)\left|\Delta_{j}\right| \rightarrow 0$; consequently, from Lemmas 3 and 4 we obtain the first assertion in (1). The fulfilment of (4.2) is obvious, since the left hand side equals to

$$
S_{I}(x \mid g)+S_{I}\left(\frac{x}{|\theta|} \mid g\right)+S_{I}\left(\frac{x}{|\theta|^{2}} \mid g\right)+\ldots
$$

If (4.1) is convergent, then so is $\prod(1 / t)\left|\Delta_{j}\right|$, and by (3.4) the second assertion follows. The proof of the last assertion is similar.

As a consequence we have

Theorem 2. Let $f \in \mathcal{A}_{\theta}$, and assume that it has a limit distribution, i.e.

$$
\lim _{x \rightarrow \infty} \frac{1}{\pi x^{2}} \#\{\alpha|| \alpha \mid \leq x, f(\alpha)<y\}=F(y)
$$

exists, where $F$ is a distribution function. Then both of the series

$$
\begin{aligned}
& \sum_{j=0}^{\infty} \sum_{c \in \mathcal{A}} f\left(c \theta^{j}\right), \\
& \sum_{j=0}^{\infty} \sum_{c \in \mathcal{A}} f^{2}\left(c \theta^{j}\right)
\end{aligned}
$$

are convergent. 
If (4.4), (4.5) are convergent, then for each interval $I \subseteq[-1 / 2,1 / 2)$, $\lim _{x \rightarrow \infty} \frac{1}{\pi x^{2}|I|} \#\{\alpha|| \alpha \mid \leq x, f(\alpha)<y, \arg \alpha \in I\}=F(y)$.

The characteristic function of $F$ can be given by

$$
\varphi(\tau)=\prod_{j=0}^{\infty}\left\{\frac{1}{t} \sum_{c \in \mathcal{A}} e^{i \tau f\left(c \theta^{j}\right)}\right\} .
$$

Another corollary of Theorem 1 is

Theorem 3. Let $f \in \mathcal{A}_{\theta}, f\left(c \theta^{j}\right)=O(1)$ as $j \rightarrow \infty, c \in \mathcal{A}$,

$$
\begin{gathered}
m_{j}=\frac{1}{t} \sum_{c \in \mathcal{A}} f\left(c \theta^{j}\right), \quad \sigma_{j}^{2}=\frac{1}{t} \sum_{c \in \mathcal{A}}\left(f\left(c \theta^{j}\right)-m_{j}\right)^{2}, \\
T_{N}^{2}:=\sum_{j=0}^{\infty} \sigma_{j}^{2}, \quad E_{N}=\sum_{j=0}^{N} m_{j} .
\end{gathered}
$$

Assume that $T_{N} \rightarrow \infty$. Let $I \subseteq[-1 / 2,1 / 2)$ be an interval. Then

$$
\frac{1}{\pi x^{2}|I|} \#\left\{\alpha|| \alpha \mid<x, \frac{\arg \alpha}{2 \pi} \in I, \frac{f(\alpha)-E_{N_{x}}}{T_{N_{x}}}<y\right\}=\left(1+o_{x}(1)\right) \Phi(y),
$$

where $\Phi$ is the Gaussian law.

Theorems 2 and 3 can be derived from Theorem 1 by making use of the method of characteristic functions in probability theory.

5. The local distribution of the sum of digits and similar additive functions. Assume that $f \in \mathcal{E}_{\theta}$, the values of $f\left(c \theta^{j}\right)(c \in \mathcal{A})$ are rational integers, and that $f\left(c \theta^{j}\right)=f(c)$ for every $j \geq 0, c \in \mathcal{A}$. Assume furthermore that the greatest common divisor of the values $\{f(c) \mid c \in \mathcal{A}\}$ is 1 .

Let $\xi_{j}(j=0,1, \ldots)$ be identically distributed independent random variables with distribution

$$
P\left(\xi_{j}=f(c)\right)=1 / t \quad(c \in \mathcal{A}) .
$$

Let $\eta_{N}=\xi_{0}+\ldots+\xi_{N-1}$ and

$$
m=\frac{1}{t} \sum_{c \in \mathcal{A}} f(c), \quad \sigma^{2}=\frac{1}{t} \sum_{c \in \mathcal{A}}(f(c)-m)^{2}, \quad \varphi(y)=\frac{1}{\sqrt{2 \pi}} \exp \left(-\frac{y^{2}}{2}\right) .
$$

According to Theorem 6 (Chapter VII) in the book of V. V. Petrov [5],

$$
\left|P\left(\eta_{N}=k\right)-\frac{1}{\sqrt{N}} \varphi\left(\frac{k-m N}{\sigma \sqrt{N}}\right)\right|=O\left(\frac{1}{N}\right)
$$

as $N \rightarrow \infty$, uniformly in $k$. 
Since $\varphi\left(\omega_{2}\right)-\varphi\left(\omega_{1}\right)=\varphi^{\prime}(\xi)\left(\omega_{2}-\omega_{1}\right)$ with some $\xi \in\left[\omega_{1}, \omega_{2}\right]$, and $\varphi^{\prime}(\xi)=$ $-\xi \varphi(\xi)$, from (5.1) we easily obtain

$$
\left|P\left(\eta_{N}=k_{1}\right)-P\left(\eta_{N}=k_{2}\right)\right| \ll \frac{\left|k_{2}-k_{1}\right|}{N} \varphi(\xi)+O\left(\frac{1}{N}\right),
$$

where $\xi$ is located in the interval with endpoints $\left(k_{i}-m N\right) /(\sigma \sqrt{N})(i=1,2)$.

We would like to count

$$
R_{k}:=\#\left\{\alpha \mid \alpha \in x C_{I}, f(\alpha)=k\right\} .
$$

Acting as in the proof of Lemmas 3 and 4 , we write each $\alpha$ as $\beta+$ $\theta^{M_{x}} \gamma, \beta \in \Gamma_{M_{x}}$. Let $\alpha \in x C_{I}, \alpha=\beta+\theta^{M_{x}} \gamma, f(\alpha)=k$. Let us drop $\alpha$ if there is some $\beta^{\prime} \in \Gamma_{M_{x}}$ for which $\beta^{\prime}+\theta^{M_{x}} \gamma \notin x C_{I}$. The cardinality of these integers is at most $O\left(x|\theta|^{M_{x}}\right)$. Fixing a remaining $\gamma$, we count those $\beta \in \Gamma_{M_{x}}$ for which $f\left(\beta+\theta^{M_{x}} \gamma\right)=k$.

The size of these numbers is

$$
t^{M_{x}} P\left(\eta_{M_{x}}=k-f\left(\theta^{M_{x}} \gamma\right)\right) .
$$

Since $|\gamma| \leq|\theta|^{N_{x}-M_{x}+1}$, the value $f\left(\theta^{M_{x}} \gamma\right)=f(\gamma)$ is bounded by $N_{x}-M_{x}+1$.

From (5.2) we see that (5.4) equals

$$
t^{M_{x}} P\left(\eta_{M_{x}}=k\right)+O\left(t^{M_{x} / 2}\right)+O\left(t^{M_{x} / 2}\left(N_{x}-M_{x}+1\right) \varphi\left(\xi_{\gamma}\right)\right)
$$

where $\xi_{\gamma}$ is located in the interval with endpoints

$$
\frac{k-m M_{x}}{\sigma \sqrt{M_{x}}}, \quad \frac{k-f(\gamma)-m M_{x}}{\sigma \sqrt{M_{x}}} .
$$

Let us sum over the appropriate values of $\gamma$, i.e. over those for which $\beta+\theta^{M_{x}} \gamma \in x C_{I}$ for every $\beta \in \Gamma_{M_{x}}$. The number of appropriate Gaussian integers $\gamma$ approximately equals the number of Gaussian integers in the annulus $x \theta^{-M_{x}} C_{I}$ with error bounded by the boundary, which is $O\left(x \cdot|\theta|^{-M_{x}}\right)$, thus it is

$$
\left(1-\frac{1}{t^{2}}\right) \pi|I| \frac{x^{2}}{t^{M_{x}}}+O\left(\frac{x}{|\theta|^{M_{x}}}\right)
$$

Since $\varphi$ is a bounded function, from (5.5) we deduce that

$$
\begin{aligned}
R_{k}= & \left(1-\frac{1}{t^{2}}\right) \pi|I| x^{2} P\left(\eta_{M_{x}}=k\right)+O\left(\frac{x^{2}}{t^{M_{x} / 2}}\right) \\
& +O\left(\left(N_{x}-M_{x}+1\right) x^{2} t^{-M_{x} / 2}\right)+O(x) .
\end{aligned}
$$

Let us choose now $M_{x}=N_{x}-\left[c \log N_{x}\right]$, with a positive constant $c$. Then the error terms on the right hand side of (5.6) are bounded by $O\left(x^{2(1-\delta)}\right)$ with some constant $\delta>0$. 
From (5.1) we easily get

$$
P\left(\eta_{N_{x}}=k\right)=P\left(\eta_{M_{x}}=k\right)+O\left(\frac{\left(\log N_{x}\right)^{3 / 2}}{N_{x}}\right)
$$

uniformly in $k$.

Let

$$
\xi_{1}=\frac{k-m N_{x}}{\sigma \sqrt{N_{x}}}, \quad \xi_{2}=\frac{k-m M_{x}}{\sigma \sqrt{M_{x}}} .
$$

If $\left|\xi_{1}\right| \geq \sqrt{\log N_{x}}$, then from (5.1) both of $P\left(\eta_{N_{x}}=k\right), P\left(\eta_{M_{x}}=k\right)$ are less than $O\left(1 / N_{x}\right)$. If $\left|\xi_{1}\right| \leq \sqrt{\log N_{x}}$, then

$$
\xi_{2}=\xi_{1}+O\left(\frac{\log N_{x}}{\sqrt{N_{x}}}\right), \quad \text { and so } \xi_{2}^{2}=\xi_{1}^{2}+O\left(\frac{\left(\log N_{x}\right)^{3 / 2}}{\sqrt{N_{x}}}\right),
$$

whence

$$
\left|e^{-\xi_{2}^{2} / 2}-e^{-\xi_{1}^{2} / 2}\right| \ll \frac{\left(\log N_{x}\right)^{3 / 2}}{\sqrt{N_{x}}} e^{-\xi_{1}^{2} / 2},
$$

and by (5.1),

$$
\begin{aligned}
\left|P\left(\xi_{N_{x}}=k\right)-P\left(\xi_{M_{x}}=k\right)\right| & \\
& \ll\left|\frac{1}{\sqrt{N_{x}}}-\frac{1}{\sqrt{M_{x}}}\right|+\frac{\left(\log N_{x}\right)^{3 / 2}}{N_{x}} \ll \frac{\left(\log N_{x}\right)^{3 / 2}}{N_{x}} .
\end{aligned}
$$

Thus

$$
R_{k}=\left(1-\frac{1}{t^{2}}\right) \pi|I| x^{2}\left\{\frac{1}{\sqrt{N_{x}}} \varphi\left(\frac{k-m N_{x}}{\sigma \sqrt{N_{x}}}\right)+O\left(\frac{\left(\log N_{x}\right)^{3 / 2}}{N_{x}}\right)\right\}
$$

We formulate our result in the following

TheOREM 4. Let $f \in \mathcal{E}_{\theta}, f\left(c \theta^{j}\right)=f(c)=$ rational integer for $c \in \mathcal{A}$, and assume that the greatest common divisor of $f(c)(c \in \mathcal{A})$ is 1 . Let

$$
m=\frac{1}{t} \sum f(c), \quad \sigma^{2}=\frac{1}{t} \sum(f(c)-m)^{2} .
$$

Then (5.8) holds for $R_{k}$ defined in (5.3).

Let $N_{I}(x \mid k)$ be the number of Gaussian integers $\alpha$ satisfying $f(\alpha)=k$ in the sector $|\alpha| \leq x,(\arg \alpha) /(2 \pi) \in I$. Then

$$
N_{I}(x \mid k)=\pi|I| x^{2}\left\{\frac{1}{\sqrt{N_{x}}} \varphi\left(\frac{k-m N_{x}}{\sigma \sqrt{N_{x}}}\right)+O\left(\frac{\left(\log N_{x}\right)^{3 / 2}}{N_{x}}\right)\right\} .
$$

Proof. It remains to prove (5.9). This follows immediately if we use (5.8) by choosing $x, x / t, x / t^{2}$ and observing that $N_{x}, N_{x / t}, \ldots$ are close to $N_{x}$.

REMARK. The sum of digits function with respect to number systems over $\mathbb{Z}[i]$ has been investigated earlier by Grabner and Liardet [2]. 


\section{References}

[1] H. Delange, Sur les fonctions q-additives ou q-multiplicatives, Acta Arith. 21 (1972), 285-298.

[2] P. J. Grabner and P. Liardet, Harmonic properties of the sum-of-digits function for complex bases, ibid. 91 (1999), 329-349.

[3] I. Kátai, Generalized number systems and fractal geometry, Pécs, Hungary, 1995 (manuscript).

[4] I. Kátai and J. Szabó, Canonical number systems for complex integers, Acta Sci. Math. (Szeged) 37 (1975), 225-260.

[5] V. V. Petrov, Sums of Independent Random Variables, Springer, Berlin, 1975.

[6] G. Steidl, On symmetric representation of Gaussian integers, BIT 29 (1989), 563571.

Computer Algebra Department

Eötvös Loránd University

Pázmány Péter sétány I/D

H-1117 Budapest, Hungary

E-mail: katai@compalg.inf.elte.hu
Université de Provence, CMI

Château Gombert

39, Rue Joliot-Curie

13453 Marseille, Cedex 13, France

E-mail: liardet@gyptis.univ.-mrs.fr

Received on 10.7.2000 